\title{
Multi-gene assay and clinical characteristics research in papillary thyroid carcinoma
}

\author{
Chang Deng ${ }^{1} \wedge$, Shan $\mathrm{Li}^{2}$, Zhixin Yang ${ }^{1}$, Yi Dou ${ }^{1}$, Daixing Hu${ }^{1}$, Jiang Zhu ${ }^{1}$, Denghui Wang ${ }^{1}$, Xinliang Su ${ }^{1 \wedge}$ \\ ${ }^{1}$ Department of Endocrine and Breast Surgery, the First Affiliated Hospital of Chongqing Medical University, Chongqing, China; ${ }^{2}$ Department of \\ Gastroenterology, the First Affiliated Hospital of Chongqing Medical University, Chongqing, China \\ Contributions: (I) Conception and design: X Su, C Deng; (II) Administrative support: X Su; (III) Provision of study materials or patients: J Zhu, Z \\ Yang, D Wang, C Deng; (IV) Collection and assembly of data: J Zhu, Z Yang, D Wang, C Deng; (V) Data analysis and interpretation: X Su, C Deng; \\ (VI) Manuscript writing: All authors; (VII) Final approval of manuscript: All authors. \\ Correspondence to: Xinliang Su. Department of Endocrine and Breast Surgery, The First Affiliated Hospital, Chongqing Medical University, 1 \\ Friendship Road, Yuzhong District, Chongqing 400016, China. Email: suxinliang@21cn.com.
}

Background: To investigate the significance of multi-gene assay in papillary thyroid carcinoma (PTC) patients in clinical practice.

Methods: From April to December 2019, medical records of 68 patients with PTC after the initial surgery were retrospectively collected and analyzed in terms of the relations between gene mutations and clinicopathological characteristics.

Results: RET/PTC rearrangement was not detected in BRAF V600E mutation patients $(\mathrm{P}<0.001)$. Besides, compared with wild-type patients, BRAF V600E mutation was associated with significantly older age $(\mathrm{P}=0.001)$ and a higher rate of extrathyroid invasion $(\mathrm{P}=0.023)$. Significantly higher BRAF V600E mutation rates were found in clinical lymph node-negative $(\mathrm{P}=0.041)$ and non-metastatic lateral lymph nodes $(\mathrm{P}=0.027)$ patients as $\mathrm{RET} / \mathrm{PTC}$ rearrangement was associated with younger age $(\mathrm{P}=0.001)$ and the increasing metastatic number of lymph nodes $(\mathrm{P}=0.020)$. Compared to other gene mutations, the multivariate analysis showed that larger tumor size [odds ratio (OR), 8.831; 95\% CI: 1.971-35.578; P=0.004], the BRAF V600E mutation alone(OR, 10.567; 95\% CI: 1.748-63.873; $\mathrm{P}=0.010)$ or in combination with one additional gene mutation (OR, 8.654; 95\% CI: 1.453-68.603; P=0.041), and Hashimoto's thyroiditis (OR, 0.112; 95\% CI: $0.025-0.499 ; \mathrm{P}=0.004)$ were all independent predictors for the prevalence of ETE.

Conclusions: BRAF V600E mutation was associated with older age and the aggressiveness of PTC but was independent of lymph node metastasis (LNM). RET/PTC rearrangement suggested more LNM in young patients with PTC. BRAF V600E mutation combined with other gene mutations, namely, multi-gene mutations, could indicate a higher aggressiveness in PTC.

Keywords: Papillary thyroid carcinoma (PTC); multi-gene assay; gene mutation

Submitted Jul 02, 2020. Accepted for publication Nov 11, 2020.

doi: $10.21037 /$ gs-20-589

View this article at: http://dx.doi.org/10.21037/gs-20-589

\section{Introduction}

Papillary thyroid carcinoma (PTC), with a 10-year survival rate over $95 \%$, is the most common type of thyroid cancer (1). Nevertheless, its recurrence rate is $9-30 \%$, according to a previous study (2-4). For patients with high-risk PTC, the poor prognosis may be related to clinical factors such as gender, extrathyroid invasion, lymph node metastasis (LNM), and distant metastasis (5).

^ORCID: Chang Deng, 0000-0002-1108-9624; Xinliang Su, 0000-0001-5792-1407. 
Currently, with the advancements in molecular biology, gene mutations have become a hot topic in thyroid carcinoma research. Increasingly many molecular markers of PTC are being used in the diagnosis and prognostic assessment. BRAF, which is the most common gene, is considered to have a highly positive predictive value for thyroid malignant tumors, with a specificity of nearly $100 \%(6,7)$. Additionally, the coexistence of BRAF and TERT promoter mutations was reported to be related to higher aggressiveness of PTC by two studies $(8,9)$. Hence, the identification of invasive PTC patients at the genomics level is of substantial significance; however, most current studies focus on the detection of a single gene or a couple of several biomarkers rather than multigene assay.

Therefore, we conducted a retrospective study to assess the association between gene mutations and clinical characteristics by using 57 gene chips of tumor pathogenesis pathways to detect the samples from the patients with PTC. We present the following article in accordance with the MDAR and STROBE reporting checklists (available at http://dx.doi.org/10.21037/gs-20-589).

\section{Methods}

The study was conducted in accordance with the Declaration of Helsinki (as revised in 2013). The study was approved by the ethics committee of The First Affiliated Hospital of Chongqing Medical University (No. 2020-220) and informed consent was taken from all the patients. From April to December 2019, medical records from PTC patients, treated at the First Affiliated Hospital of Chongqing Medical University were retrospectively collected. Preoperatively, physical examination, ultrasonography, fibrolaryngoscopy, and thyroid function examination were conducted.

Patients who were diagnosed with PTC pathologically after initial operations were included in the study. Consequently, the pathological results included extrathyroidal extension (ETE) information, and the number of metastatic lymph nodes (LNMN) was extracted. The exclusion criteria were as follows: (I) history of head or neck irradiation; (II) family history of thyroid tumor; (III) patients without a non-PTC histology result; (IV) reoperation; (V) the disagreement of gene testing; and (VII) incomplete clinical data. Then, informed patient consent was obtained from the included patients before molecular tests were performed.

The core part of PTC specimens was stored in a frozen tube, which was handled by a company, namely, USCI (Beijing Youxun Medical Laboratory Co., Ltd.). An Illumina NextSeq 500 high-throughput sequencer was used for sequencing. The remaining tissue was fixed to $10 \%$ formaldehyde and sent to the pathological diagnosis center for diagnosis by two pathologists.

The TNM stages were identified according to the 8th edition of the American Joint Committee on Cancer (AJCC) (10). The risk stratification of recurrence was divided according to the 2015 American Thyroid Association (ATA) guidelines (1).

\section{Next-generation sequencing}

Genomic DNA was isolated from fresh tumor tissues or FFPE using a QIAamp DNA FFPE Tissue Kit (Qiagen) according to the manufacturer's instructions. The concentration of the DNA was determined using a Qubit fluorometer 3.0 (Life Technologies). A custom-designed 2.2 Mb panel, which covered exons and partial introns of cancer driver genes, hereditary cancer-related genes, and therapy-related genes, was used in this study. Then, 50$100 \mathrm{ng}$ of sheared genomic DNA was subjected to library construction with an MGIEasy universal DNA library kit (MGI, China), which was followed by hybrid capture using an $\mathrm{xGen}$ Hybridization and Wash Kit (IDT, USA). Libraries' quality and concentration were determined using a LabChip ${ }^{\circledR}$ GX Touch $^{\mathrm{TM}}$ nucleic acid analyzer (PerkinElmer, USA) and a Qubit fluorometer 3.0 (Life Technologies, USA), respectively. Tumor-matched normal samples were also sequenced as controls. The qualified libraries were sequenced with $2 \times 100$ bp paired-end reads on an MGISEQ-2000 (MGI, China) platform.

\section{Bioinformatic analysis}

The paired-end reads were mapped to the hg19/GRCh37 reference using BWA (v 0.7.12)-MEM. SNVs and InDels were called by VarScan (v 2.4.3) by verified settings. CNVkit was used to identify copy number variants. SNVs and InDels from tissue and plasma were filtered by depths $>1,000 \times$ and $400 \times$, respectively. Gene amplification in tissue and plasma were defined as depths $>900 \times$ and $1,000 \times$ respectively, and $\mathrm{CN}$ values called by $\mathrm{CNVkit}$ were higher than 4 . 


\section{Statistical analysis}

SPSS 25.0 statistical software was used for all analyses. Continuous data were expressed as the mean \pm SD and analyzed via the independent $t$-test. Categorical data were expressed as a percentage (\%) and analyzed via Pearson's $\chi^{2}$ test or Fisher's exact test. Univariate and multivariate analyses were conducted in our study. A two-tailed $\mathrm{P}$ value of $<0.05$ was regarded as statistically significant.

\section{Results}

\section{Demographic features of PTC patients}

A total of 68 patients were included in our study, with a mean age (years) of $38.9 \pm 11.3$, and $51(75 \%)$ patients were female. Besides, 33 (48.5\%) patients were clinically lymph node-negative (cN0), of which $24(72.7 \%)$ cases had confirmed LNM. The total LNM rate was $82.4 \%(56 / 68)$ and $38(55.9 \%)$ patients had more than 5 LNMs. Moreover, $53(77.9 \%)$ patients had central lymph node metastasis (CLNM), and 39 (57.4\%) patients had lateral lymph node metastasis (LLNM). Additional information on the patients is presented in Table 1.

\section{Results}

Sixty-eight PTC samples were collected for multi-gene assay. The most common mutation type was BRAF, which

Table 1 The association of BRAF V600E, RET mutations and clinicopathologic characteristics in papillary thyroid carcinoma (case, \%)

\begin{tabular}{|c|c|c|c|c|c|c|}
\hline \multirow{2}{*}{ Items } & \multicolumn{3}{|c|}{ BRAF V600E } & \multicolumn{3}{|c|}{ RET } \\
\hline & Mutation $(n=55)$ & wild-type $(n=13)$ & $P$ value & Mutation $(n=7)$ & Wild-type $(n=61)$ & $P$ value \\
\hline Sex & & & & & & 0.670 \\
\hline Male & $16(29.1 \%)$ & $1(7.7 \%)$ & 0.160 & $1(14.3 \%)$ & $16(26.2 \%)$ & \\
\hline Female & 39 (70.9\%) & $12(92.3 \%)$ & & $6(85.7 \%)$ & 45 (73.8\%) & \\
\hline \multicolumn{7}{|c|}{ Age at diagnosis, years } \\
\hline Mean \pm SD & $40.9 \pm 10.2$ & $30.2 \pm 11.9$ & 0.001 & $25.6 \pm 12.4$ & $40.4 \pm 10.2$ & 0.001 \\
\hline$<55$ & $50(90.9 \%)$ & $13(100.0 \%)$ & 0.575 & 7 (100.0\%) & $56(91.8 \%)$ & 1.000 \\
\hline$\geq 55$ & $5(9.1 \%)$ & $0(0.0 \%)$ & & $0(0.0 \%)$ & $5(8.2 \%)$ & \\
\hline \multicolumn{7}{|c|}{ Tumor size in mm } \\
\hline Mean \pm SD & $15.1 \pm 7.6$ & $19.8 \pm 11.5$ & 0.186 & $17.7 \pm 12.0$ & $15.8 \pm 8.2$ & 0.585 \\
\hline$\leq 10$ & 18 (32.7\%) & 3 (23.1\%) & 0.740 & 3 (42.9\%) & $18(29.5 \%)$ & 0.668 \\
\hline$>10$ & 37 (67.3\%) & $10(77.9 \%)$ & & $4(57.1 \%)$ & $43(70.5 \%)$ & \\
\hline cNO & & & & & & 0.107 \\
\hline Yes & 30 (54.5\%) & $3(23.1 \%)$ & 0.041 & $1(14.3 \%)$ & 32 (52.5\%) & \\
\hline No & $25(45.5 \%)$ & 10 (76.9\%) & & $6(85.7 \%)$ & $29(47.5 \%)$ & \\
\hline Location & & & & & & 0.409 \\
\hline Upper & $20(36.4 \%)$ & $3(23.1 \%)$ & 0.519 & $1(14.3 \%)$ & $22(36.1 \%)$ & \\
\hline Middle/lower & $35(63.6 \%)$ & $10(76.9 \%)$ & & $6(85.7 \%)$ & 39 (63.9\%) & \\
\hline HT & & & & & & 0.390 \\
\hline Yes & $14(25.5 \%)$ & 5 (38.5\%) & 0.492 & 3 (42.9\%) & $16(26.2 \%)$ & \\
\hline No & 41 (74.5\%) & 8 (61.5\%) & & $4(57.1 \%)$ & 45 (73.8\%) & \\
\hline
\end{tabular}

Table 1 (continued) 
Table 1 (continued)

\begin{tabular}{|c|c|c|c|c|c|c|}
\hline Items & \multicolumn{3}{|c|}{ BRAF V600E } & \multicolumn{3}{|c|}{ RET } \\
\hline ETE & & & & & & 0.415 \\
\hline Yes & 38 (69.1\%) & $4(30.8 \%)$ & 0.023 & 3 (42.9\%) & 39 (63.9\%) & \\
\hline No & 17 (30.9\%) & 9 (69.2\%) & & $4(57.1 \%)$ & $22(36.1 \%)$ & \\
\hline Yes & $9(16.4 \%)$ & $2(15.4 \%)$ & 1.000 & $1(14.3 \%)$ & $10(16.4 \%)$ & \\
\hline No & 46 (83.6\%) & $11(84.6 \%)$ & & $6(85.7 \%)$ & $51(83.6 \%)$ & \\
\hline \multicolumn{7}{|l|}{ LNMN } \\
\hline Mean \pm SD & $6.7 \pm 6.7$ & $10.2 \pm 7.0$ & 0.090 & $13.0 \pm 5.8$ & $6.7 \pm 6.7$ & 0.020 \\
\hline CLNM & & & & & & 1.000 \\
\hline Yes & 42 (76.4\%) & $11(84.6 \%)$ & 0.717 & $6(85.7 \%)$ & 47 (77.0\%) & \\
\hline No & $13(23.6 \%)$ & $2(15.4 \%)$ & & $1(14.3 \%)$ & $14(23.0 \%)$ & \\
\hline LLNM & & & & & & 0.225 \\
\hline Yes & 28 (50.9\%) & $11(84.6 \%)$ & 0.027 & $6(85.7 \%)$ & 33 (54.1\%) & \\
\hline No & 27 (49.1\%) & $2(15.4 \%)$ & & $1(14.3 \%)$ & $28(45.9 \%)$ & \\
\hline Stage & & & & & & 1.000 \\
\hline I/II & 53 (96.4\%) & 13 (100.0\%) & 1.000 & 7 (100.0\%) & 59 (96.7\%) & \\
\hline
\end{tabular}

cNO, clinical lymph node-negative; HT, Hashimoto's thyroiditis; ETE, extrathyroidal extension; LNMN, the Metastatic number of lymph nodes; CLNM, central lymph node metastasis; LLNM, lateral lymph node metastasis.

accounted for $80.9 \%$, and all the BRAF mutations were of type BRAF V600E, while 7 patients (10.3\%) harbored RET/PTC rearrangements and 3 cases were free from any mutations (see Figure 1). Notably, among these 55 cases of BRAF V600E mutation, 20 were in combination with other gene mutations, of which 14 patients had two gene mutations and 6 patients had three gene mutations (see Figure 1).

\section{Relationship between BRAF V600E and other gene mutations}

There were $55(80.9 \%)$ cases of BRAF mutations in all genetic events, and 7 (10.3\%) cases of RET/PTC rearrangements. No RET/PTC rearrangement events were identified in patients with BRAF mutations $(\mathrm{P}<0.001$, Table 2). 


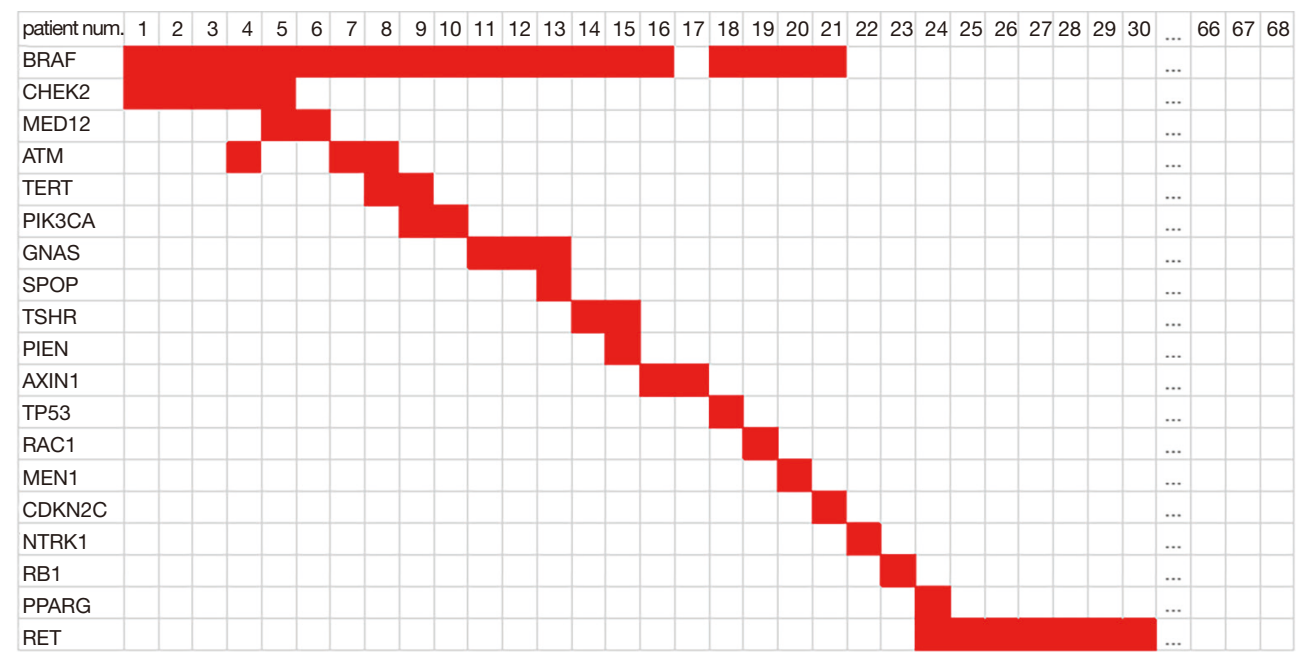

Figure 1 Distribution of gene mutations in papillary thyroid carcinoma. The omitted part represents that 35 patients with BRAF V600E mutation alone are not shown in the figure. The red part expresses that patient with the corresponded gene mutation. The blank part means no mutation. num., number; BRAF, B-Raf proto-oncogene, serine/threonine kinase; CHEK2, checkpoint kinase 2; MED12, mediator complex subunit 12; ATM, ATM serine/threonine kinase; TERT, telomerase reverse transcriptase; PIK3CA, phosphatidylinositol-4,5bisphosphate 3-kinase, catalytic subunit alpha; GNAS, guanine nucleotide-binding protein, $\alpha$-stimulating complex locus; SPOP, speckletype POZ protein; TSHR, thyroid stimulating hormone receptor; PTEN, phosphatase and tensin homolog; AXIN1, axin 1; TP53, tumor protein p53; RAC1, ras-related C3 botulinum toxin substrate 1; MEN1, multiple endocrine neoplasia 1; CDKN2C, cyclin-dependent kinase inhibitor 2C; NTPK1, neurotrophic tyrosine kinase, receptor, type 1; RB1, retinoblastoma 1; PPARG, peroxisome proliferator-activated receptor gamma; RET, rearranged during transfection.

Table 2 The association of BRAF V600E mutation and other gene mutations (case, \%)

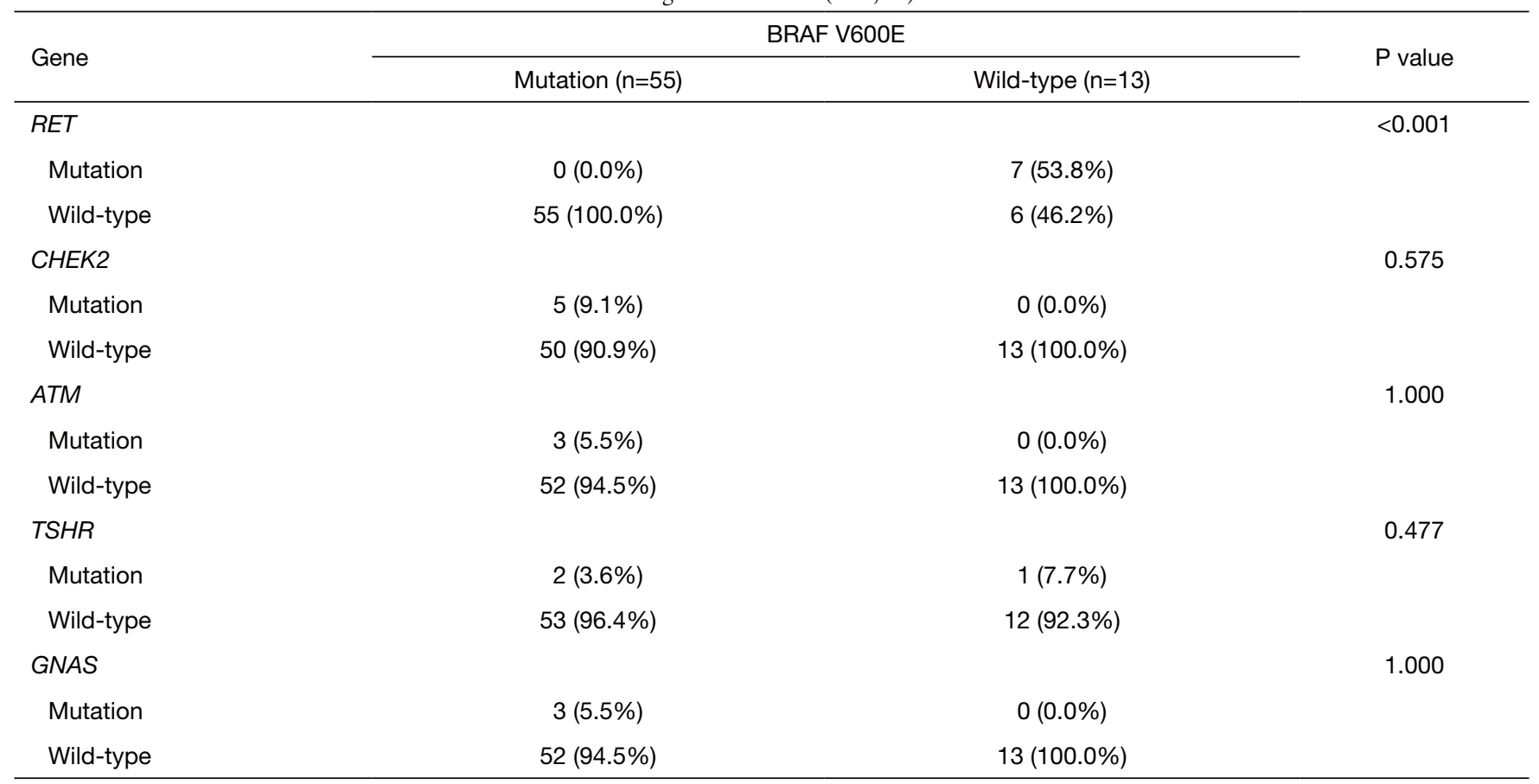

BRAF, B-Raf proto-oncogene, serine/threonine kinase; RET, rearranged during transfection; CHEK2, checkpoint kinase 2; ATM, ATM serine/threonine kinase; TSHR, thyroid-stimulating hormone receptor; GNAS, guanine nucleotide-binding protein, $\alpha$-stimulating complex locus. 
Table 3 The association between ETE and clinicopathological characteristics or gene mutations in PTC

\begin{tabular}{|c|c|c|c|c|}
\hline Characteristics & \multicolumn{2}{|c|}{ Univariate analysis } & \multicolumn{2}{|c|}{ Multivariate analysis } \\
\hline Sex (female vs. male) & $0.595(0.182-1.943)$ & 0.390 & NA & NA \\
\hline Age ( $\geq 55$ vs. $<55$ years) & $0.380(0.040-3.601)$ & 0.399 & NA & NA \\
\hline Tumor size (>1 vs. $\leq 1 \mathrm{~cm}$ ) & $5.833(1.904-17.808)$ & 0.002 & $8.831(1.971-35.578)$ & 0.004 \\
\hline Multifocality (yes vs. no) & $1.429(0.388-5.258)$ & 0.592 & NA & NA \\
\hline LNMN ( $\geq 5$ vs. $<5)$ & $0.888(0.331-2.382)$ & 0.813 & NA & NA \\
\hline RET mutation (yes vs. no) & $0.423(0.870-2.065)$ & 0.288 & NA & NA \\
\hline BRAF mutations only* & $4.313(1.097-16.955)$ & 0.036 & $10.567(1.748-63.873)$ & 0.010 \\
\hline
\end{tabular}

${ }^{*}$ Comparisons were performed between BRAF mutation groups (BRAF mutations only, BRAF + one gene mutations and BRAF + two gene mutations) and non-BRAF mutation group. ETE, extrathyroidal extension; PTC, papillary thyroid carcinoma; OR, odds ratio; Cl, confidence interval; HT, Hashimoto's thyroiditis; LNMN, the Metastatic number of lymph nodes.

\section{Association between the BRAF V600E mutation or RET/ PTC rearrangement and clinicopathological characteristics}

Compared with wild-type BRAF, the BRAF V600E mutation was associated with older age $(\mathrm{P}=0.001$, Table 1) and extrathyroid invasion $(\mathrm{P}=0.023)$. Interestingly, the cN0 group $(\mathrm{P}=0.041)$ and lateral lymph node-negative group ( $\mathrm{P}=0.027)$ both had higher BRAF V600E mutation rates. The patients in the RET/PTC rearrangement group were of younger age $(\mathrm{P}=0.001)$ and showed more LNMs $(\mathrm{P}=0.020)$. More importantly, RET/PTC rearrangement was deemed to be related to higher rates of LNMN exceeding $5(\mathrm{P}=0.015)$. No significant difference in other clinical factors was identified.

\section{Association between ETE and clinicopathological characteristics or gene mutations in PTC}

The univariate analysis showed that the presence of ETE was associated with larger tumor size $(\mathrm{P}=0.002$, Table 3), Hashimoto's thyroiditis (HT) $(\mathrm{P}<0.001)$, the BRAF V600E mutation alone $(\mathrm{P}=0.036)$, and two gene mutations $(\mathrm{P}=0.017)$, while multivariate analyses showed that larger tumor size [odds ratio (OR), 8.831; 95\% CI: 1.971-35.578; $\mathrm{P}=0.004]$, the BRAF V600E mutation alone (OR, 10.567; 95\% CI: 1.748-63.873; $\mathrm{P}=0.010)$ and two gene mutations (OR, 8.654; 95\% CI: 1.453-68.603; P=0.041) were all independent predictors for a high prevalence of ETE. Compared to other gene mutations group, the three gene mutations group, combined with BRAF V600E mutation, tended to have a higher risk of ETE, although it was not statistically significant $(\mathrm{P}=0.082)$. However, $\mathrm{HT}(\mathrm{OR}, 0.112$; 95\% CI: 0.025-0.499; $\mathrm{P}=0.004)$ may be a protective factor against ETE.

\section{Discussion}

BRAF encodes a serine-threonine-specific kinase on chromosome $7 \mathrm{q} 34$. BRAF V600E is the most frequent mutation site in differentiated thyroid cancer, which accounts for approximately $90 \%$ of mutations (11), and the BRAF protein plays a role in regulating the activity of the mitogen-activated protein kinase (MAPK) to promote PTC cell proliferation and differentiation $(12,13)$. Studies have reported that preoperative fine-needle aspiration cytology (FNAC) in combination with BRAF and other gene tests can reduce unnecessary surgery for patients who have been diagnosed with "uncertain" thyroid nodules and can provide a foundation for assessing the prognosis of PTC $(6,14)$.

In our study, the BRAF mutation frequency was $80.9 \%$, higher than generally reported $45-80.8 \%(11,15-18)$, and all the mutation sites were located at the 600th base, which could be explained by the similarity of the included patients in terms of region and ethnicity. There is no doubt that the 
BRAF mutation can be used as a diagnostic biomarker, but its use for prognosis assessment is controversial. Studies have identified relationships between BRAF mutations and LNM, extracapsular invasion, vascular invasion, advanced age, tumor size, aggressive subtype, recurrence, and death, among other factors (18-20). However, a Japanese study yielded the opposite result (21). This study also demonstrated that patients with BRAF mutations were more likely to be older and to exhibit ETE. Nevertheless, in terms of LNM, there was no correlation between LNMN or CLNM and BRAF mutation. Surprisingly, in our study, in contrast to ETE, less LLNM was observed in patients with BRAF mutations. These contradictory results might cast doubt on the prognostic assessment value of BRAF mutations. No relationship or negative correlation between LNM and ETE is identified, which needs future studies to prove. In summary, we inferred that BRAF mutation alone would not be an excellent biomarker for prognosis.

Moreover, the current study found that $\mathrm{cN} 0$ patients had a higher BRAF mutation rate, which was likely because the current $\mathrm{cN} 0$ standard is not sufficiently accurate. The cN0 patients, who account for $48.5 \%$ of the patients, were shown to have an LNM rate of $72.7 \%$, which exceeds the rate that was reported in our previous study (22). Therefore, for PTC patients with occult LNM, preoperative routine evaluation is not sufficient. Our institution can guide intraoperative frozen biopsy of central lymph node dissection for clinical decisions (23), but it requires time and money. From there, a further prospective study of multi-gene sequencing to identify cases with a high risk of recurrence and invasion in well-differentiated thyroid cancer is urgently needed.

Furthermore, the RET gene is a proto-oncogene on chromosome $10 \mathrm{q} 11.2$, and it acts as a tyrosine protease receptor. Besides, the mechanism that occurs in PTC is gene fusion, which is known as RET/PTC rearrangement (24). $\mathrm{RET} / \mathrm{PTC} 1$ and RET/PTC3 are the most common types, in which RET is fused with CCDC6 and NCOA4, respectively. The frequency of RET mutations is second only to that of BRAF mutations in PTC (25), and Sapio et al. also identified RET/PTC rearrangement in benign lesions, which is related to the high growth rate (26). Hence, RET/PTC rearrangement cannot be used as a unique molecular marker of PTC for diagnosis. We found that the RET/PTC rearrangement rate was $10.3 \%$, but none of these rearrangements had a BRAF mutation, which was consistent with the results of Soares et al. studies (27). To the best of our knowledge, PTC frequently has genetic alterations that lead to the activation of the MAPK signaling pathway, which includes RET/PTC rearrangement and point mutations of the BRAF and RAS genes. Consequently, these two gene mutations may be exclusive or alternative mutations in the etiopathogenesis of PTC.

In the progression of PTC, young age or a history of radiation exposure may be related to $\mathrm{RET} / \mathrm{PTC}$ rearrangement $(24,28)$. Similar results are obtained in this study; hence, RET/PTC rearrangement may correspond to a higher risk of recurrence or poor prognosis in PTC, especially in young patients. None of our patients had been exposed to radiation, from which we inferred that radiation exposure may not contribute to the increased incidence of PTC. Additionally, we did not identify a correlation between RET/PTC rearrangement and tumor aggressiveness, e.g., manifested as ETE or increased LNM, which differs from the results of by Romei et al. (29). Considering the heterogeneity between the studies, it is speculated that a single gene mutation may not affect the prognosis of PTC patients.

Ito et al. found that age was an independent risk factor for lymph node recurrence (2). However, the current PTC risk stratification of recurrence, based on clinicopathological factors, is often insufficient to accurately identify the highrisk PTC patients. We found that BRAF mutation was more common in older people, but RET/PTC rearrangement was more common in young patients. Gene mutations of different ages may play an important role in PTC. Therefore, we suggest that the future risk stratification systems incorporate molecular markers in association with age, as has been newly reported (30).

BRAF and TERT promoter mutations were the most common mutations in the current study. Several previous studies have shown that the combination of BRAF and TERT promoter mutations suggested a high aggressiveness and a high risk of recurrence of PTC $(8,9,20,31)$, while our previous studies found that both of them were related to PTC invasiveness, except LNM (32). It is reasonable to suspect that the simultaneous presence of two gene mutations is a useful predictor of LNM.

Due to the low prevalence of the TERT promoter and other gene mutations in our study, no relevant statistical analysis was conducted. We analyzed the associations of ETE, which was associated with tumor recurrence and patient mortality (5), with clinicopathological characteristics and gene mutations. ETE plays an important role in the current PTC risk stratification of recurrence, which was defined as the invasion of a tumor beyond the thyroid capsule into adjacent tissues. Previous studies showed that 
the BRAF V600E mutation was related to ETE and LNM (18-20). However, no studies that identified the number of biomarkers with ETE in PTC have been reported. Compared to other gene events, we found that the more gene mutations that are based on BRAF V600E mutation are identified, the higher the prevalence of ETE. The three gene mutation groups corresponded to an $\sim 11$-fold higher risk of ETE, although no statistical significance was found, which could be due to the small sample size effect. In summary, our study demonstrated that BRAF V600E in combination with other gene mutations could predict a poor prognosis, which may guide our future exploration.

Furthermore, Hashimoto's thyroiditis, which is also known as chronic lymphocytic thyroiditis, was regarded as a risk factor for the development of PTC. However, we found that HT could be a protective predictor against ETE, which was consistent with the mainstream reported view $(33,34)$. Moreover, no relation was identified between $\mathrm{LNMN} \geq 5$ and ETE, and both of these factors were considered in the criteria for the risk stratification of recurrence. Though the effect of HT on the prognosis of PTC remains controversial and the mechanism of HT in the development of ETE has yet to be elucidated, we supposed that the presence of ETE with HT does not influence the biological behavior (e.g., LNM) of PTC, which was also reported by other studies $(35,36)$.

Due to the limited sample size, we failed to identify biomarker panels for PTC, such as the 21 genetic markers that are used to evaluate the risk of recurrence of breast cancer (37), for the identification of aggressive PTC phenotypes for more aggressive treatment options, and it was difficult to draw a waterfall plot in this study. Nevertheless, we remain dedicated to the identification of improved biomarker panels, which will be a future direction for our further study.

\section{Conclusions}

PTC had a high BRAF mutation rate, and BRAF mutations were related to tumor aggressiveness but not to LNM. RET/PTC rearrangement may be a biomarker for LNM in young PTC patients, although it is relatively rare. Furthermore, the multi-gene mutation that is based on the BRAF V600E mutation could predict the poor prognosis in PTC patients.

\section{Acknowledgments}

Funding: None.

\section{Footnote}

Reporting Checklist: The authors have completed the MDAR and STROBE reporting checklists. Available at http:// dx.doi.org/10.21037/gs-20-589

Data Sharing Statement: Available at http://dx.doi. org/10.21037/gs-20-589

Peer Review File: Available at http://dx.doi.org/10.21037/gs20-589

Conflicts of Interest: All authors have completed the ICMJE uniform disclosure form (available at http://dx.doi. org/10.21037/gs-20-589). The authors have no conflicts of interest to declare.

Ethical Statement: The authors are accountable for all aspects of the work in ensuring that questions related to the accuracy or integrity of any part of the work are appropriately investigated and resolved. The study was conducted in accordance with the Declaration of Helsinki (as revised in 2013). The study was approved by the ethics committee of The First Affiliated Hospital of Chongqing Medical University (No. 2020-220) and informed consent was taken from all the patients.

Open Access Statement: This is an Open Access article distributed in accordance with the Creative Commons Attribution-NonCommercial-NoDerivs 4.0 International License (CC BY-NC-ND 4.0), which permits the noncommercial replication and distribution of the article with the strict proviso that no changes or edits are made and the original work is properly cited (including links to both the formal publication through the relevant DOI and the license). See: https://creativecommons.org/licenses/by-nc-nd/4.0/.

\section{References}

1. Haugen BR, Alexander EK, Bible KC, et al. 2015 American Thyroid Association Management Guidelines for Adult Patients with Thyroid Nodules and Differentiated Thyroid Cancer: The American Thyroid Association Guidelines Task Force on Thyroid Nodules and Differentiated Thyroid Cancer. Thyroid 2016;26:1-133.

2. Ito Y, Miya A, Kudo T, et al. Prognostic factors for recurrence of papillary thyroid carcinoma in the lymph nodes, lung, and bone: analysis of 5,768 patients with 
average 10-year follow-up. World J Surg 2012;36:1274-8.

3. Shaha AR. Recurrent differentiated thyroid cancer. Endocr Pract 2012;18:600-3.

4. Hartl DM, Mamelle E, Borget I, et al. Influence of prophylactic neck dissection on rate of retreatment for papillary thyroid carcinoma. World J Surg 2013;37:1951-8.

5. Guo K, Wang Z. Risk factors influencing the recurrence of papillary thyroid carcinoma: a systematic review and metaanalysis. Int J Clin Exp Pathol 2014;7:5393-403.

6. Jinih M, Foley N, Osho O, et al. BRAF(V600E) mutation as a predictor of thyroid malignancy in indeterminate nodules: A systematic review and meta-analysis. Eur J Surg Oncol 2017;43:1219-27.

7. Zhang M, Zhang Y, Fan X, et al. Thyroid cytological examination by using fine needle aspiration biopsy combined with BRAF gene testing: its diagnostic value in differentiating benign from malignant thyroid nodules. J Interventronal Radiol 2017;26:622-6.

8. Xing M, Liu R, Liu X, et al. BRAF V600E and TERT promoter mutations cooperatively identify the most aggressive papillary thyroid cancer with highest recurrence. J Clin Oncol 2014;32:2718-26.

9. Jin L, Chen E, Dong S, et al. BRAF and TERT promoter mutations in the aggressiveness of papillary thyroid carcinoma: a study of 653 patients. Oncotarget 2016;7:18346-55.

10. Lamartina L, Grani G, Arvat E, et al. 8th edition of the AJCC/TNM staging system of thyroid cancer: what to expect (ITCO\#2). Endocr Relat Cancer 2018;25:L7-L11.

11. Lee SE, Hwang TS, Choi YL, et al. Molecular Profiling of Papillary Thyroid Carcinoma in Korea with a High Prevalence of BRAFV600E Mutation. Thyroid 2017;27:802-10.

12. Nikiforov YE. Thyroid carcinoma: molecular pathways and therapeutic targets. Mod Pathol 2008;21 Suppl 2:S37-43.

13. LiVolsi VA. Papillary thyroid carcinoma: an update. Mod Pathol 2011;24:S1-S9.

14. Tian W, Yao J. Paying attention to the standardization of diagnosis and treatment of thyroid nodules. Chin J Pract Surg 2015;35:579-83.

15. Tufano RP, Teixeira GV, Bishop J, et al. BRAF Mutation in Papillary Thyroid Cancer and Its Value in Tailoring Initial Treatment. Medicine 2012;91:274-86.

16. Ke Z, Liu Y, Zhang Y, et al. Diagnostic value and lymph node metastasis prediction of a custom-made panel (thyroline) in thyroid cancer. Oncol Rep 2018;40:659-68.

17. Hong AR, Lim JA, Kim TH, et al. The Frequency and
Clinical Implications of the BRAFV600EMutation in Papillary Thyroid Cancer Patients in Korea Over the Past Two Decades. Endocrinol Metab (Seoul) 2014;29:505-13.

18. Liang J, Cai W, Feng D, et al. Genetic landscape of papillary thyroid carcinoma in the Chinese population. J Pathol 2018;244:215-26.

19. Elisei R, Ugolini C, Viola D, et al. BRAF(V600E) mutation and outcome of patients with papillary thyroid carcinoma: a 15-year median follow-up study. J Clin Endocrinol Metab 2008;93:3943-9.

20. Xing M, Alzahrani AS, Carson KA, et al. Association Between BRAF V600E Mutation and Mortality in Patients With Papillary Thyroid Cancer. JAMA 2013;309:1493-501.

21. Nasirden A, Akaike K, Tomomasa R, et al. In Japanese patients with papillary thyroid carcinoma, TERT promoter mutation is associated with poor prognosis, in contrast to BRAF (V600E) mutation. Virchows Arch 2016;469:687-96.

22. Sun R, Pan X, Su X, et al. Characteristics related to lymph node metastasis and strategy of lymph node dissection in papillary thyroid carcinoma. China Oncol 2016;26:80-7.

23. Hu D, Zhou J, He W, et al. Risk factors of lateral lymph node metastasis in $\mathrm{cN} 0$ papillary thyroid carcinoma. World J Surg Oncol 2018;16:30.

24. Santoro M, Carlomagno F. Central role of RET in thyroid cancer. Cold Spring Harb Perspect Biol 2013;5:a009233.

25. Wei J, Wang Y, Sun X, et al. Research progress in thyroid cancer related genes and their applications in clinical diagnosis. Chin J Pract Surg 2018;44:880-5.

26. Sapio MR, Guerra A, Marotta V, et al. High growth rate of benign thyroid nodules bearing RET/PTC rearrangements. J Clin Endocrinol Metab 2011;96:E916-9.

27. Soares P, Trovisco V, Rocha AS, et al. BRAF mutations and RET/PTC rearrangements are alternative events in the etiopathogenesis of PTC. Oncogene 2003;22:4578-80.

28. Nikiforov YE, Nikiforova MN. Molecular genetics and diagnosis of thyroid cancer. Nat Rev Endocrinol 2011;7:569-80.

29. Romei C, Ciampi R, Elisei R. A comprehensive overview of the role of the RET proto-oncogene in thyroid carcinoma. Nat Rev Endocrinol 2016;12:192-202.

30. Subash A, Sinha P, Singh A. BRAF mutation and age in differentiated thyroid cancer risk stratification: Two sides of the same coin. Oral Oncol 2020;106:104732.

31. Liu R, Bishop J, Zhu G, et al. Mortality Risk Stratification by Combining BRAF V600E and TERT Promoter Mutations in Papillary Thyroid Cancer: Genetic Duet of 
BRAF and TERT Promoter Mutations in Thyroid Cancer Mortality. JAMA Oncol 2017;3:202-8.

32. Ren H, Shen Y,Hu D, et al. Co-existence of BRAF(V600E) and TERT promoter mutations in papillary thyroid carcinoma is associated with tumor aggressiveness, but not with lymph node metastasis. Cancer Manag Res 2018;10:1005-13.

33. Lee JH, Kim Y, Choi JW, et al. The association between papillary thyroid carcinoma and histologically proven Hashimoto's thyroiditis: a meta-analysis. Eur J Endocrinol 2013;168:343-9.

34. Huang BY, Hseuh C, Chao TC, et al. Well-differentiated thyroid carcinoma with concomitant Hashimoto's

Cite this article as: Deng C, Li S, Yang Z, Dou Y, Hu D, Zhu J, Wang D, Su X. Multi-gene assay and clinical characteristics research in papillary thyroid carcinoma. Gland Surg 2021;10(1):242-251. doi: 10.21037/gs-20-589 thyroiditis present with less aggressive clinical stage and low recurrence. Endocr Pathol 2011;22:144-9.

35. Furlan JC, Bedard YC, Rosen IB. Significance of tumor capsular invasion in well-differentiated thyroid carcinomas. Am Surg 2007;73:484-91.

36. Lee I, Kim HK, Soh EY, et al. The Association Between Chronic Lymphocytic Thyroiditis and the Progress of Papillary Thyroid Cancer. World J Surg 2020;44:1506-13.

37. Dinan MA, Mi X, Reed SD, et al. Initial Trends in the Use of the 21-Gene Recurrence Score Assay for Patients With Breast Cancer in the Medicare Population, 2005-2009.

JAMA Oncol 2015;1:158-66. 